\title{
Half-plane contacts subject to remote tension
}

\author{
Nils Cwiekala and David A Hills
}

\begin{abstract}
The state of stress present in an elastic half-plane contact problem, where one or both bodies is subject to remote tension has been investigated, both for conditions of full stick and partial slip. The state of stress present near the contact edges is studied for different loading scenarios in an asymptotic form. This is of practical relevance to the study of contacts experiencing fretting fatigue, and enables the environment in which cracks nucleate to be specified.
\end{abstract}

\author{
Keywords \\ Bulk tension, half-plane contact, partial slip, fretting fatigue, asymptotes
}

Date received: 6 August 2020; accepted: 21 December 2020

\section{Introduction}

Fretting and fretting fatigue are important problems arising in gas turbines and a wide range of mechanical assemblies - in fact wherever there are components in notionally stationary contact but where the applied loads or vibration cause very small amounts of differential motion between the contacting surfaces and which consequently may cause damage promoting cracks to nucleate. Of course it is the presence of shear tractions, $q(x)$, which induces slip and, for elastically similar bodies, this may have two origins - the application of a shear force $Q$ (Figure 1(a)) or the presence of a differential tension $\sigma_{0}=\sigma_{1}-\sigma_{2}$ acting parallel with the surface, Figure 1(b). Unless the tensions parallel with the surface are equal in each body shear tractions will certainly develop, and which may add to or subtract from the effect of shear force, depending on which edge of the contact is being studied.

In some problems, such as the standard laboratory test for fretting fatigue strength, Figure 1(d), it is very easy to identify the bulk tension present in each component (here, none in the pad and simply the tension in the dogbone specimen) but in many cases it is very much harder to isolate the effect of tension, such as in the fanblade dovetail arrangement in Figure 1(e), with the blade being subject to a centrifugal and vibrational load, $F_{\mathrm{c}}$ and $F_{\mathrm{v}}$, respectively. Andresen et al. ${ }^{1}$ have recently looked at means of identifying the effective bulk tension present, and there is little doubt that it has a first order effect on both the magnitude of slip and also on the state of stress present in each body. Here we concentrate on the latter, and look at contacts represented by half-planes being a typical approximation for incomplete contacts. ${ }^{1}$ These contacts typically arise when bodies with convex surfaces are pressed against each other by a normal load, and show the characteristic that the contact area increases in size with an increasing normal load. In the standard laboratory test (Figure 1(d)) as well as the fanblade dovetail arrangement (Figure 1(e)) we find this type of contact. We study the differences for the application of a differential tension compared to the application of a shear load, and how the combination of $\sigma_{1}$ and $\sigma_{2}$ affects the state of stress around the edge of the contact.

We start from the assumption that the coefficient of friction, $f$, is sufficiently high to prevent all slip at the interface. If all slip is inhibited, the problem is equivalent to an infinite plane with two semi-infinite cracks indented into it from $\pm \infty$ to $\pm a$, Figure 1(b), and we start from the case where the upper half-plane $(y>0)$ is free of remote loads while the lower is subjected to remote tension $\sigma_{1}=\sigma_{0}$. It has been shown, ${ }^{2}$ using the

Department of Engineering Science, University of Oxford, Oxford, UK

\section{Corresponding author:}

Nils Cwiekala, Department of Engineering Science, University of Oxford, Parks Road, Oxford, Oxfordshire OXI 3PJ, UK.

Email: nils.cwiekala@eng.ox.ac.uk 


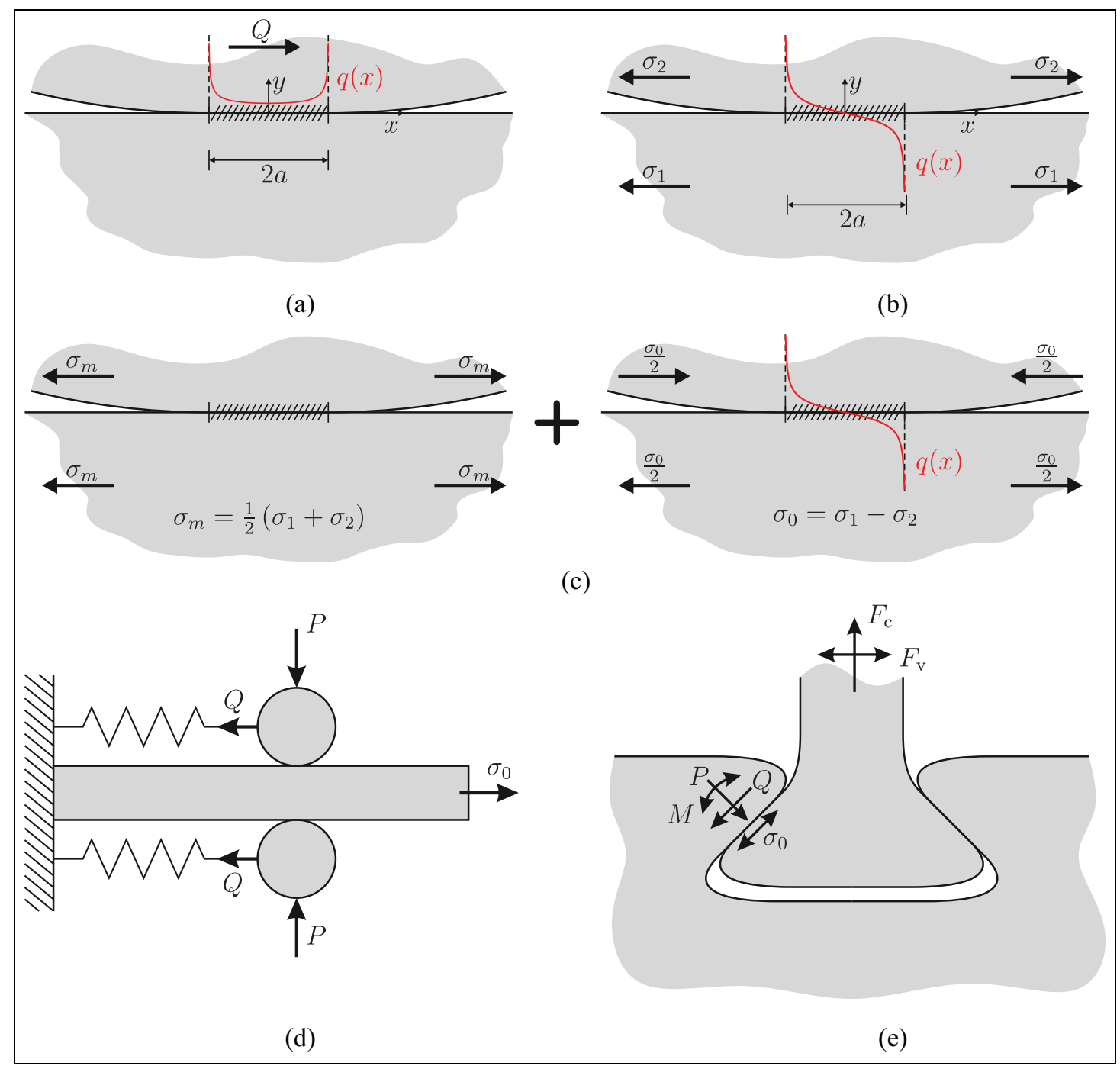

Figure I. Shear traction in different contact settings: (a) shear traction $q(x)$ resulting from tangential load $Q$, (b) shear traction resulting from differential bulk tension $\sigma_{0}$, both for incomplete, fully stuck contacts, (c) representation of arbitrary combinations of $\sigma_{1}$ and $\sigma_{2}$ by superposition of equal tension (magnitude and sign) in both bodies and equal (magnitude) but opposite tension, (d) schematic represantation of standard fretting fatigue test, ${ }^{3}$ and (e) Schematic represantation of dovetail arrangement subject to external loads. ${ }^{4}$

Mossakovskii-Barber approach, that the traction arising along the interface, $y=0$ and $|x|<a$, is given by

$$
\sigma_{x y}(x, 0)=q(x)=-\frac{\sigma_{0}}{4} \frac{x}{\sqrt{a^{2}-x^{2}}}
$$

and the state of stress in the body is therefore the sum of the state of stress induced in a half-plane by tractions (1) applied along the surface together with, for the lower body only, uniform remote tension $\sigma_{0}$.

\section{State of stress present}

If we consider only the half-plane $y<0$ and exert tractions (1) over the surface the Muskhelishvili complex potential is given by, ${ }^{5}$

$$
\begin{aligned}
\phi(z) & =\frac{1}{2 \pi} \int_{\text {Contact }} \frac{\sigma_{x y}(x, 0)}{x-z} d x \\
& =-\frac{\sigma_{0}}{8 \pi} \int_{-a}^{a} \frac{x\left(a^{2}-x^{2}\right)^{-\frac{1}{2}}}{x-z} d x \\
& = \begin{cases}-\frac{\sigma_{0}}{8}\left(1+\frac{z}{\sqrt{z^{2}-a^{2}}}\right) & x<0 \\
-\frac{\sigma_{0}}{8}\left(1-\frac{z}{\sqrt{z^{2}-a^{2}}}\right) & x \geqslant 0\end{cases}
\end{aligned}
$$

where $z=x+i y, i=\sqrt{-1}$.

The same result is obtained without the need for any integration by making use of the relationship ${ }^{6}$

$$
\phi(z)=-\frac{\lambda}{2}-\frac{1}{2} i \sigma_{x y}^{a d}(z)
$$




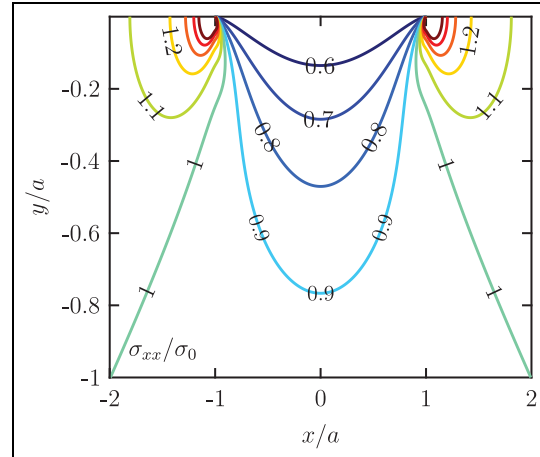

(a)

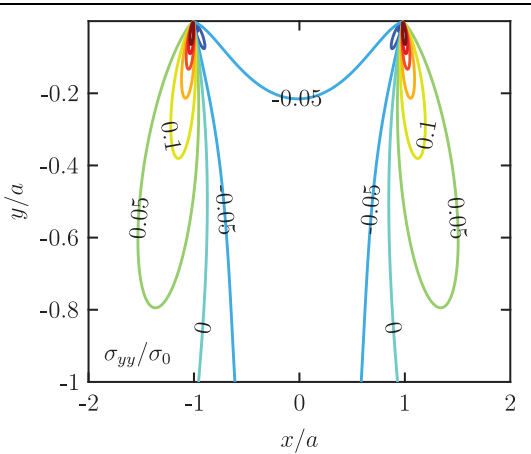

(b)

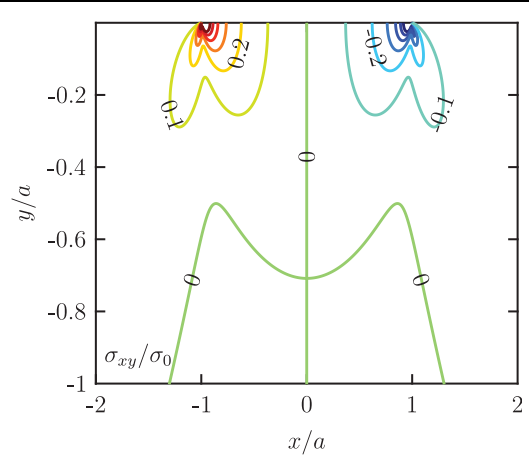

(c)

Figure 2. Subsurface stress field for fully stuck contact with differential bulk tension, $\sigma_{0}$, applied to the lower body only: (a) normalised stress $\sigma_{x x} / \sigma_{0}$, (b) normalised stress $\sigma_{y y} / \sigma_{0}$, and (c) normalised stress $\sigma_{x y} / \sigma_{0}$.

where $\sigma_{x y}^{a d}$ is the shear stress inside the stick zone. The quantity $\lambda$ is a load case dependent parameter defined by the singular integral equation

$$
\frac{1}{\pi} \int_{\text {Contact }} \frac{\sigma_{x y}(\xi, 0)}{x-\xi} d \xi=\lambda \quad \forall x \in \text { stick zone }
$$

where the Cauchy principal value must be considered. For a differential bulk tension $\sigma_{0}, \lambda=\sigma_{0} / 4$, independent of the individual components $\sigma_{1}$ and $\sigma_{2}$. Here, relation (3) simply provides a different route to the same result obtained in equation (2), but later on when looking at contacts in partial slip with a comparatively complicated form of the resulting shear traction it provides a much simpler way of obtaining the complex potential compared to the evaluation of the integral over the contact interface. Independent of how the complex potential $\phi(z)$ is obtained, the corresponding state of stress is given by ${ }^{7}$

$$
\begin{aligned}
& \sigma_{x x}(x, y)=2(\phi(z)+\phi(\bar{z}))+i y\left(\frac{\partial}{\partial z} \phi(z)-\frac{\partial}{\partial \bar{z}} \phi(\bar{z})\right), \\
& \sigma_{y y}(x, y)=-i y\left(\frac{\partial}{\partial z} \phi(z)-\frac{\partial}{\partial \bar{z}} \phi(\bar{z})\right) \\
& \sigma_{x y}(x, y)=i(\phi(z)-\phi(\bar{z}))-y\left(\frac{\partial}{\partial z} \phi(z)+\frac{\partial}{\partial \bar{z}} \phi(\bar{z})\right) .
\end{aligned}
$$

Explicit expressions in real algebra can be found by separation of real and imaginary part of the potential function to $\phi(z)=u(x, y)+i v(x, y) .^{7}$ The components of stress are then obtained from the complex potential (2) and in the half-plane $y<0$ are

$$
\begin{gathered}
\sigma_{x x}(x, y)=-\frac{\sigma_{0}}{2} \mp \frac{\sigma_{0}}{8} \frac{4 c_{1} x^{2}\left(x^{2}+y^{2}+\sqrt{c_{1}}\right)}{c_{1} x \sqrt{2 c_{1}\left(\sqrt{c_{1}}+c_{2}\right)}} \\
\mp \frac{\sigma_{0}}{8} \frac{a^{2}\left(-c_{1} c_{2}+c_{2}^{3}-4 x^{2}\left(c_{1}+y^{2}\left(\sqrt{c_{1}}+c_{2}\right)\right)\right)}{c_{1} x \sqrt{2 c_{1}\left(\sqrt{c_{1}}+c_{2}\right)}}
\end{gathered}
$$

$$
\begin{gathered}
\sigma_{y y}(x, y)= \pm \frac{\sigma_{0}}{16} \frac{a^{2}}{c_{1}^{2}} \frac{1}{x} \sqrt{2 c_{1}\left(\sqrt{c_{1}}+c_{2}\right)} \\
\left(c_{2}\left(c_{2}-\sqrt{c_{1}}\right)-4 x^{2} y^{2}\right), \\
\sigma_{x y}(x, y)=\mp \frac{\sigma_{0}}{4} \frac{y c_{1}\left(x^{2}+y^{2}-\sqrt{c_{1}}\right)}{c_{1} \sqrt{2 c_{1}\left(\sqrt{c_{1}}+c_{2}\right)}} \\
\mp \frac{\sigma_{0}}{4} \frac{y a^{2} c_{2}\left(\sqrt{c_{1}}+2 c_{2}\right)}{c_{1} \sqrt{2 c_{1}\left(\sqrt{c_{1}}+c_{2}\right)}},
\end{gathered}
$$

with $\quad c_{1}=\left((a+x)^{2}+y^{2}\right)\left((a-x)^{2}+y^{2}\right) \quad$ and $c_{2}=x^{2}-y^{2}-a^{2}$. The main steps of the derivation are given in Appendix 1. With the upper signs corresponding to $x<0$ and the lower signs to $x \geqslant 0$, expressions (6) describe the state of stress induced by tractions (1). We will refer to this solution as the contact stress field in what follows. For the full stress field in the lower body $(y<0)$ we need to superimpose the remote bulk tension $\sigma_{1}$. We note that if we want to express the remotely applied stresses $\sigma_{1}$ and $\sigma_{2}$ by their difference $\sigma_{0}$ and their mean $\sigma_{m}=\frac{1}{2}\left(\sigma_{1}+\sigma_{2}\right)$, then the contact stress field depends on the difference only, while the full stress field depends on the mean as well.

The full subsurface stress field for the load case represented by Figure 1(b) with $\sigma_{1}=\sigma_{0}$ and $\sigma_{2}=0$ is shown in Figure 2. As expected $\sigma_{y y}$ and $\sigma_{x y}$ vanish if we move sufficiently far away from the contact. The stress parallel to the surface $\sigma_{x x}$ tends to $\sigma_{0}$ remote from the contact and to $\sigma_{0} / 2=\sigma_{m}$ within the contact.

If the tension in each body is the same in magnitude and sign the presence of the interface has no effect and there is a uniform $\sigma_{x x}$ stress everywhere. Note that, generally if a tension $\sigma_{1}$ is present in one body and $\sigma_{2}$ in the other body (Figure 3(b)) the solution may be found by superposition of these two solutions viz a mean stress of $\frac{1}{2}\left(\sigma_{1}+\sigma_{2}\right)$, everywhere, together with stresses of $\pm \frac{1}{2}\left(\sigma_{1}-\sigma_{2}\right)$ applied to the two bonded half-planes (Figure 3(c)).

The special case of tension applied to one body and an equal compression in the other, both of magnitude 


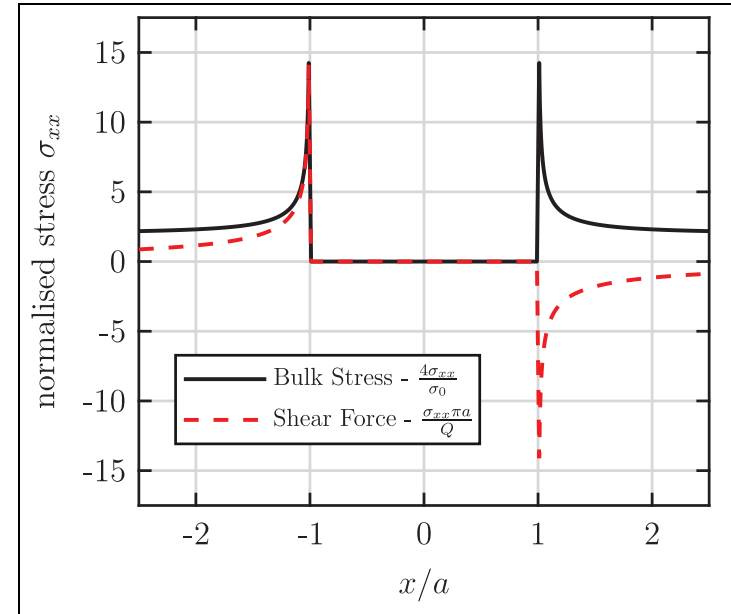

(a)

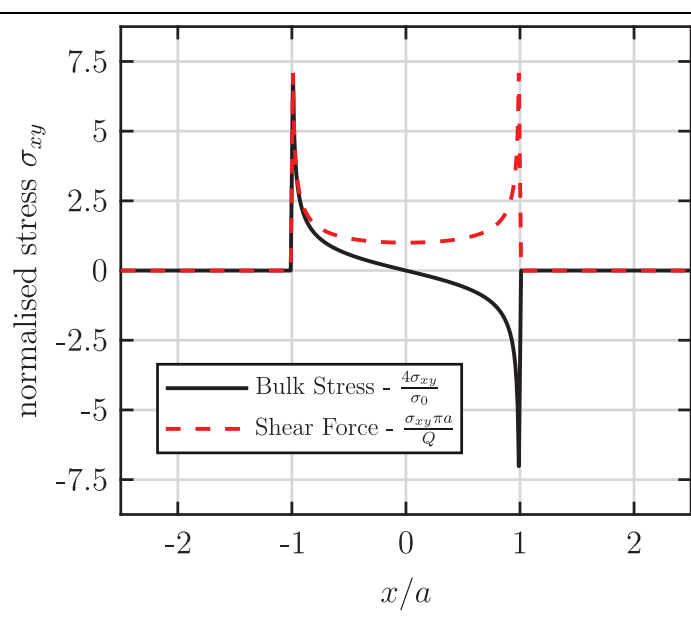

(b)

Figure 3. Non-vanishing components of stress along the contact interface for the application of equal and opposite remote stress $\sigma_{0} / 2$ (solid) and the application of a shear load (dashed) corresponding to Figure I (a): (a) normalised stress $\sigma_{x x}$ and (b) normalised stress $\sigma_{x y}$.

$\sigma_{0} / 2\left(\sigma_{m}=0\right)$, may also be treated by the relations found and the non-vanishing components of the full stress field along the surface $(y \rightarrow 0)$ are shown in Figure 3. We see that for the case of equal and opposite loading, as $\sigma_{m}=0$, the stress parallel to the surface vanishes along the interface, that is, on $y=0,|x|<a$. At points remote to the contact it tends to $\sigma_{1}=\sigma_{0} / 2$.

Furthermore, we use Figure 3 to compare the stress along the contact interface, $y=0$, to the stress present for the application of a shear load as shown in Figure 1(a). We used different normalisations for both load cases to ensure that the shear stress shows the same severity in the singularity when approaching the edge of the contact (Figure 3(b)). We note from Figure 3(a) that even though the stress parallel to the surface does vanish for both load cases inside the contact, $|x|<a$, and the resulting singularity in the stress parallel to the interface is similar for both load cases, the stress will differ remote from the contact.

\section{Asymptotic solutions}

In the previous section we examined the state of stress in adhered half-plane contacts subject to remote tension, in detail, and compared some results with the stress state induced by the application of a shear force. One reason for doing this was as a prelude to understanding how the different forms of load affect the behaviour of contact edges, under adhered conditions and in the presence of slip. So far we considered the stress inside the contact region, $|x|<a$ and remote from the contact, $|x| \gg a$, but not the singularity at the edge of the contact.

We have had some success in representing the state of stress near an incomplete contact edge in asymptotic form. The contact pressure is locally square root bounded but, if the contact is adhered the shear tractions induced are, as we have just seen, square root singular in form. One important aspect of the asymptotic representation which has been missing is an understanding of the asymptotic field when the shear tractions are excited by bulk tension; information is lost when only the surface tractions induced are considered, and here we derive the local solution when tension induced, first under conditions of full stick. If we move the origin to the left hand edge by the transformation $s=x+a$ equation (1) becomes

$$
\begin{aligned}
\sigma_{x y}(s) & =-\frac{\sigma_{0}}{4} \frac{s-a}{\sqrt{2 a s-s^{2}}} \\
& \approx K_{I I} \frac{1}{\sqrt{s}} \quad \text { for } \quad s \ll a
\end{aligned}
$$

where $K_{I I}=\sigma_{0} \sqrt{a} / 4 \sqrt{2}{ }^{8}$ To find the stress parallel to the surface or the subsurface stress field in an asymptotic description we can proceed in one of two ways. Either we start from the negative branch of the finite potential function (2) and again apply a shift of the origin with $w=z+a$ followed by a binomial expansion as well as the corresponding simplifications to the derivation of the asymptotic contact shear traction which yields

$$
\begin{aligned}
\phi(w) & =-\frac{\sigma_{0}}{8}\left(1+\frac{w-a}{\sqrt{w^{2}-2 a w}}\right) \\
\approx & \frac{K_{I I}}{2} \frac{1}{\sqrt{-w}}-\frac{\sigma_{0}}{8} \quad \text { for } \quad \operatorname{abs}(w) \ll a .
\end{aligned}
$$

The different steps with corresponding assumptions for the derivation of equations (7) and (8) are given in 
Appendix 2. For the potential function the same result can be obtained by using the asymptotic expression of the shear traction (7) in equation (3) and choosing the appropriate branch of the function $\pm \sqrt{w}$

$$
\begin{aligned}
\phi(w) & =-\frac{\lambda}{2}-\frac{1}{2} i \sigma_{x y}^{a d}(w) \\
& =-\frac{\sigma_{0}}{8}+\frac{1}{2} i K_{I I} \frac{1}{\sqrt{w}} \\
& =\frac{K_{I I}}{2} \frac{1}{\sqrt{-w}}-\frac{\sigma_{0}}{8} .
\end{aligned}
$$

The resulting potential function differs from the potential function for the application of a shear load by the constant term $-\sigma_{0} / 8$ only. From equation (5) we can immediately see that a constant real term in the potential function will contribute to the stress parallel to the surface only and we find $\sigma_{x x}=-\sigma_{0} / 2$. The complete stress field includes the superposition of the remotely applied tension $\sigma_{1}$ (for the lower body). In the neighbourhood of the contact edge the stress parallel to the surface then differs by $-\sigma_{0} / 2+\sigma_{1}=\sigma_{m}$ for the two load cases. All other components of stress are unchanged, that is, the shear force induced $K_{I I}$ solution and the bulk stress induced $K_{I I}$ solution are the same. We note that for the special case of an equal tension and compression in the two bodies, that is, $\sigma_{m}=0$ as considered before, the whole stress field in the neighbourhood of the contact edge will be the same as when the stress is excited by a shear force.

\section{Effect of slip}

We now want to investigate the effect of a differential bulk stress on the subsurface stress field by allowing for local slip at the contact interface of an incomplete contact. We consider, here, a finite Hertzian contact with the known contact pressure

$$
\sigma_{y y}(x, 0)=p(x)=-\frac{p_{0}}{a} \sqrt{a^{2}-x^{2}} \text { for }|x| \leqslant a,
$$

where $p_{0}$ is the peak contact pressure. After the application of the normal load we apply a bulk tension $\sigma_{0}$ to the lower body. Symmetric zones of slip will establish themselves at the edges of the contact. The resulting shear traction was found by Nowell and Hills, ${ }^{9}$ Ciavarella and Macina ${ }^{10}$ and Moore et al. ${ }^{11}$ Moore et al. use distributed dislocations along the contact interface to account for relative displacements of the two bodies and found the side condition for the determination of the stick-zone half-width, $c$, generally, as

$$
-\int_{c}^{a} \frac{p(s) d s}{\sqrt{s^{2}-c^{2}}}=\frac{\pi \sigma_{0}}{8 f},
$$

where $f$ is the coefficient of frizction, as before. If the Hertzian pressure distribution (10) is substituted into (11), Ciavarella and Macina's ${ }^{10}$ implicit expression

$$
K(k)-E(k)=\frac{\pi \sigma_{0}}{8 f p_{0}}
$$

is recovered, where $K(k)$ and $E(k)$ represent the complete elliptic integral of the first and second kind respectively with $k$ being the elliptic modulus (Note that there are different conventions for the notation of elliptic integrals in terms of the elliptic modulus $k$ or the parameter $m=k^{2}$.). In equation (12) and the following

$$
k=\sqrt{1-\left(\frac{c}{a}\right)^{2}} .
$$

The shear traction inside the stick zone is then given by, ${ }^{10}$

$$
\begin{gathered}
\sigma_{x y}(x, 0)=\frac{2}{\pi} \frac{f p_{0} x}{a^{2}} \sqrt{c^{2}-x^{2}}[\Pi(n, k)-K(k)] \\
\text { for }|x|<c
\end{gathered}
$$

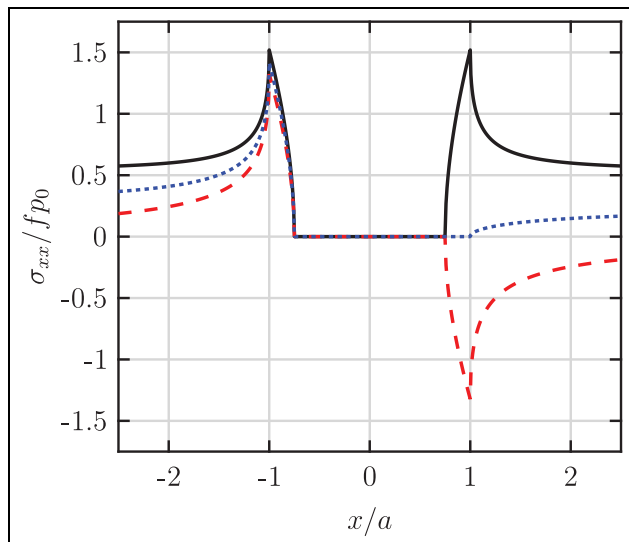

(a)

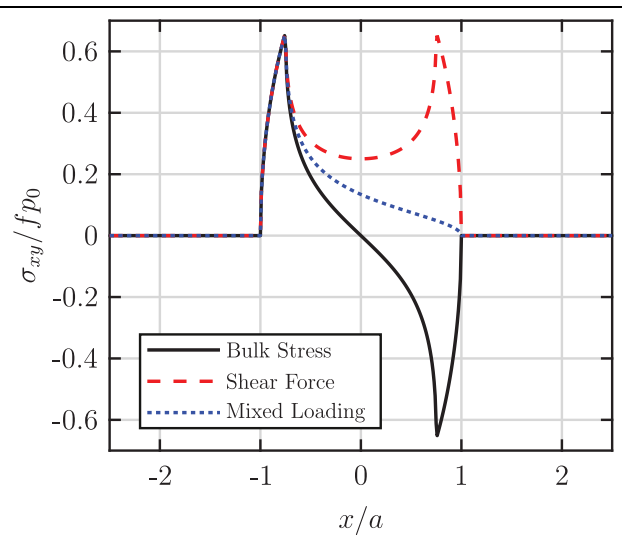

(b)

Figure 4. Non-vanishing components of stress along the contact interface for the application of equal and opposite remote stress $\sigma_{0}$ (solid), the application of a shear load (dashed) and mixed loading such that the contact is fully stuck at the leading edge (dotted) and a slip zone side of $d / a=0.25$ at the trailing edge: (a) normalised stress $\sigma_{x x}$ and (b) normalised stress $\sigma_{x y}$. 


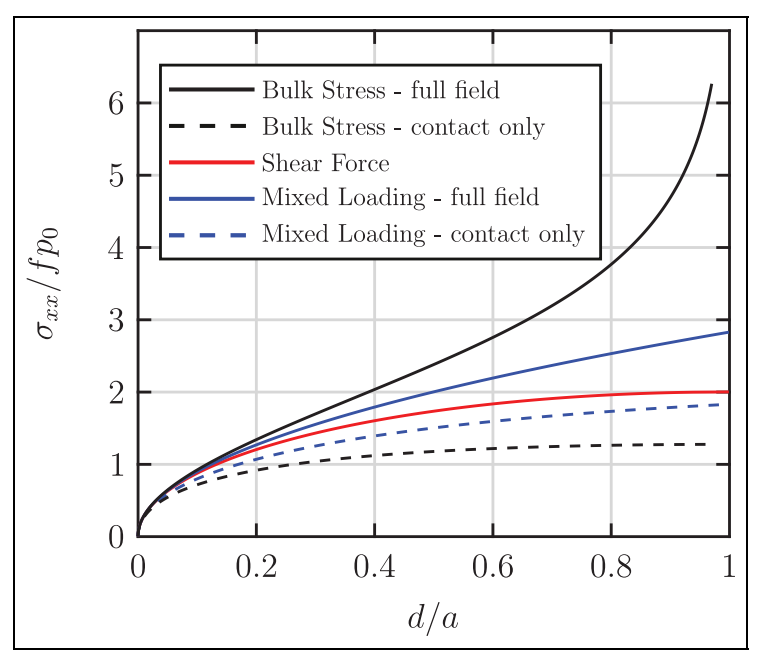

Figure 5. Maximum stress parallel to the surface plotted over the slip zone size at the left hand edge of the contact.

where $\Pi(n, k)$ represents the complete elliptic integral of the third kind, $k$ is again given by equation (13) and

$$
n=\sqrt{\frac{a^{2}-c^{2}}{a^{2}-x^{2}}}
$$

is the so called characteristic of $\Pi$. The complex potential is then simply derived by substitution of equation (14) in expression (3), where $\lambda=\sigma / 4$ as for the fully stuck case, giving

$$
\phi(z)=-\frac{\sigma_{0}}{8} \pm \frac{2}{\pi} \frac{f p_{0} z}{a^{2}} \sqrt{z^{2}-c^{2}}[\Pi(n, k)-K(k)] .
$$

Again, the upper sign in equation (16) corresponds to $x<0$ and the lower sign to $x>0$. For the case of an equal tension and compression of magnitude $\sigma_{0} / 2$ in the two bodies, the non-vanishing stresses along the contact interface are shown in Figure 4. As for the fully stuck case the stress parallel to the surface vanishes inside the stick region and has its maximum at the edge of and exterior to the contact.

We compare the results for the case of an equal tension and compression in the two bodies to the stresses resulting from two different load cases, one where we apply a shear load to the upper body and a second where we have a mix of differential bulk tension and shear load such that one edge of the contact (the right hand end) remains stuck. In each case the slip zone, $d=a-c$, at the left hand edge is of the same size and the slip displacement is in the same direction. Figure 4(b) shows the resulting shear stress which is similar for all three cases within the slip zone at the left hand edge. We are now able to compare the maximum stress parallel to the surface for the three load cases under consideration, as this is most important in terms of fretting damage. For the assumed slip zone size of $d / a=0.25$ we see that the stress will be higher if the relative displacement is caused by a bulk tension compared to a shear force (Figure 4(a)). Figure 5 shows the stress parallel to the interface at the edge of the contact not only for the slip-zone size of $d / a=0.25$, considered before, but for a continuous slip zone size.

For a slip zone size of $d / a \rightarrow 1$ and the application of a shear load we recover the result of $\sigma_{x x} / f p_{0}=2$ for a sliding contact ${ }^{12}$ while the consideration of the contact stress field alone with the application of a differential bulk tension leads to $\sigma_{x x} / f p_{0}=4 / \pi$ as shown by Ciavarella and Macina. ${ }^{10}$ Of course the results for the full stress field are somewhat arbitrary, in the case of differential bulk tension and mixed loading, since the same slip zone size could originate from different combinations of applied bulk tension and compression in the two bodies, but the stress inside the stick zone will vanish only, for the special case of equal remote tension and compression. We find from Figure 5 that the difference in the maximum stress parallel to the surface vanishes for $d \ll a$ and we will look at this in more detail by considering the asymptotic description.

\section{Asymptotic solutions}

Again, the subsurface stress-field might be described in an asymptotic formulation. It is worth noting that by representing each of the elliptical integrals in equation (12) by the first two terms of the corresponding series expansion around the edge of the contact, that is, $c / a=1$, we recover the known asymptotic expression ${ }^{13}$

$$
\begin{aligned}
& \frac{\pi \sigma_{0}}{8 f p_{0}}=K(k)-E(k)=\frac{\pi}{2}\left(1-\frac{c}{a}\right) \\
\Leftrightarrow & \frac{\sigma_{0} a}{4 f p_{0}}=a-c \\
\Leftrightarrow & \frac{2 K_{I I}}{f L_{I}}=d
\end{aligned}
$$

for the slip zone $d$ where the shear traction results from a tangential force and the asymptotic multiplier $L_{I}$ is defined as

$$
L_{I}=\lim _{s \rightarrow 0} \frac{p(s)}{\sqrt{s}} .
$$

Furthermore, for an asymptotic representation of the bilateral contact tractions with $q(s)=K_{I I} / \sqrt{s}$ (cf. equation (7)) and $p(s)=L_{I} \sqrt{s}$, Moore et al. ${ }^{11}$ derive the dislocation density to (Note that equation (29) in the original publication ${ }^{11}$ is a factor of -2 wrong.)

$$
B_{x}(s)=-\frac{\kappa+1}{2 \mu} f L_{I} \sqrt{d-s},
$$

where $\mu$ is the shear modulus of the contacting bodies and $\kappa=3-4 \nu$ is Kolosov's constant for plane strain with $\nu$ being Poisson's ratio. The resulting shear traction inside the stick zone is then given by 




Figure 6. Normalised $x x$-component, $\sigma_{x x} / f_{0}$, of the finite full stress field (solid lines) and percentage mismatch to the asymptotic stress field (dashed lines) for $d / a=0.1$.

$$
\begin{aligned}
\sigma_{x y}(x, 0) & =\frac{2 \mu}{\pi(\kappa+1)} \frac{1}{\sqrt{s}} \int_{0}^{d} \frac{\sqrt{\xi} B_{x}(\xi)}{\xi-s} d \xi \\
& =f L_{I}(\sqrt{s}-\sqrt{s-d}) \text { for } d \leqslant s
\end{aligned}
$$

and is therefore similar to the case where the shear traction originates from a tangential force $Q .{ }^{13}$ The contact stress field in the neighbourhood of the contact edge in terms of the complex potential is obtained by substitution of equation (20) into equation (3) leading to

$$
\begin{aligned}
\phi(w) & =-\frac{\lambda}{2}-\frac{1}{2} i \sigma_{x y}^{a d}(w) \\
& =-\frac{\sigma_{0}}{8}-\frac{1}{2} i f L_{I}(\sqrt{w}-\sqrt{w-d}) \\
& =-\frac{f L_{I}}{2}(\sqrt{-w}-\sqrt{-w+d})-\frac{\sigma_{0}}{8} .
\end{aligned}
$$

This complex potential for the asymptotic representation of the contact stress field is basically recovering the results obtained by Dini et al. ${ }^{14}$ but in a much more straightforward manner. We see that it differs from the stress field, associated with the application of a shear load, by a constant stress $\sigma_{x x}=-\sigma_{0} / 2$ parallel to the surface, as it does for the fully stuck case (provided that the slip zones $d$ are of equal size).

The full stress field for the lower body is then obtained by superposition of the remotely applied stress $\sigma_{1}$ and following the same arguments as for the fully stuck case we see that the full stress field in the asymptotic representation differs by the constant term $\sigma_{x x}=\sigma_{m}$ to the stress field associated with the application of a shear load and consequently they match for the special case of equal tension and compression in the two bodies (with $\sigma_{m}=0$ ).

Figure 6 shows the normalised $x x$-component, $\sigma_{x x} / f p_{0}$, of the finite full stress field around the left slip zone in the lower body for the application of an equal tension, $\sigma_{1}=\sigma_{0} / 2$, and compression, $\sigma_{2}=-\sigma_{0} / 2$ (solid lines) obtained from the finite potential (16). The shown stress field is characteristic for a relative slip zone size $d / a=0.1\left(\sigma_{0} / f p_{0}=0.41\right.$ from equation (12)). The dashed lines represent the percentage mismatch $100\left(\sigma_{x x}^{\text {finite }}-\sigma_{x x}^{\text {asympt. }}\right) / \sigma_{x x}^{\text {finite }}$ between the finite and the asymptotic description of the stress field. It is shown that the agreement is within $10 \%$ for a depth down to $0.8 d$ and generally the error decreases when approaching the slip region at the contact interface.

\section{Conclusion}

The state of stress in an incomplete contact pair induced by differential remote tensions has been explored for the cases of infinite friction (no slip) and finite friction, and for the cases of contacts of finite size, such as shown in Figure 1(a) and (b), as well as an asymptotic description of the contact edge. The differences between the state of stress induced, generally, and with particular attention to the near edge, have been investigated. It is found that, to within the accuracy of a first order description of the contact edge there is no difference in the local components $\sigma_{y y}$ and $\sigma_{x y}$. The stress acting parallel with the free surface, and which is responsible for the early propagation of any crack which may form, differs by the mean value of applied bulk tensions $\sigma_{m}=\frac{1}{2}\left(\sigma_{1}+\sigma_{2}\right)$. The only scenario where the local stress parallel to the surface is similar regardless whether the local shear tractions originate from a shear force or a differential bulk tension, is the special case of an equal tension and compression of the two bodies, that is, $\sigma_{m}=0$. Furthermore, the state of stress close to the contact edge depends on the mean value of applied bulk tensions, $\sigma_{m}$, so that a comparison of the stress field in which cracks nucleate in the fanblade dovetail arrangement (Figure 1(e)) and in the standard laboratory test (Figure 1(d)) depends not only on $\sigma_{0}$ but also $\sigma_{m}$.

\section{Declaration of conflicting interests}

The author(s) declared no potential conflicts of interest with respect to the research, authorship, and/or publication of this article.

\section{Funding}

The author(s) disclosed receipt of the following financial support for the research, authorship, and/or publication of this article: This work was supported by Rolls-Royce plc and the Engineering and Physical Sciences Research Council under the Prosperity Partnership Grant 'Cornerstone: Mechanical Engineering Science to Enable Aero Propulsion Futures' [EP/R004951/1].

\section{ORCID iD}

Nils Cwiekala (iD https://orcid.org/0000-0002-5116-756X 


\section{References}

1. Andresen H, Hills D and Moore M. Representation of incomplete contact problems by half-planes. Eur J Mech A Solids 2020; 85: 104138.

2. Hills D, Davies M and Barber J. An incremental formulation for half-plane contact problems subject to varying normal load, shear, and tension. J Strain Anal Eng Des 2011; 46(6): 436-443.

3. Nowell D. An analysis of fretting fatigue. PhD Thesis, University of Oxford, 1988.

4. Andresen H, Hills DA and Vazquez J. Closed-form solutions for tilted three-part piecewise-quadratic half-plane contacts. Int J Mech Sci 2019; 150: 127-134.

5. Muskhelishvili N. Some fundamental problems in the mathematical theory of elasticity [in russian], izd.-vo akad. Moscow: Nauk SSSR, 1964.

6. Vázquez J, Navarro C and Domínguez J. A new method for obtaining the stress field in plane contacts. Int $J$ Solids Struct 2012; 49(26): 3659-3665.

7. Vázquez J, Navarro C and Domínguez J. Explicit equations for sub-surface stress field in plane contacts. Int $J$ Mech Sci 2013; 67: 53-58.

8. Hills D, Fleury R and Dini D. Partial slip incomplete contacts under constant normal load and subject to periodic loading. Int J Mech Sci 2016; 108: 115-121.

9. Nowell D and Hills D. Mechanics of fretting fatigue tests. Int J Mech Sci 1987; 29(5): 355-365.

10. Ciavarella $M$ and Macina G. New results for the frettinginduced stress concentration on hertzian and flat rounded contacts. Int J Mech Sci 2003; 45(3): 449-467.

11. Moore M, Ramesh R, Hills D, et al. Half-plane partial slip contact problems with a constant normal load subject to a shear force and differential bulk tension. $J$ Mech Phys Solids 2018; 118: 245-253.

12. Hills D and Nowell D. Mechanics of Fretting Fatigue. Solid mechanics and its applications. Netherlands: Springer, 1994.

13. Dini D and Hills DA. Bounded asymptotic solutions for incomplete contacts in partial slip. Int $J$ Solids Struct 2004; 41(24-25): 7049-7062.

14. Dini D, Sackfield A and Hills D. Comprehensive bounded asymptotic solutions for incomplete contacts in partial slip. J Mech Phys Solids 2005; 53(2): 437-454.

15. Vázquez J, Navarro $C$ and Domínguez J. Explicit equations for the half-plane sub-surface stress field under a flat rounded contact. J Strain Anal Eng Des 2014; 49(8): 562 570.

\section{Appendix I}

Vázquez et al. ${ }^{7}$ describe how to derive the stress in terms of the real variables $(\mathrm{x}, \mathrm{y})$. In the case considered here the complex potential, $\phi(z)$, is defined by means of the complex function $f(z)=z / \sqrt{z^{2}-a^{2}}$ and we need to find a split into a real and an imaginary part $f(z)=u(x, y)+i v(x, y)$ so that

$$
\begin{aligned}
\phi(z) & =-\frac{\sigma_{0}}{8}\left(1+\frac{z}{\sqrt{z^{2}-a^{2}}}\right) \\
& =-\frac{\sigma_{0}}{8}(1+f(z)) \\
& =-\frac{\sigma_{0}}{8}-\frac{\sigma_{0}}{8} u(x, y)-i \frac{\sigma_{0}}{8} v(x, y) .
\end{aligned}
$$

For $f(z)=z / \sqrt{z^{2}-a^{2}}$ we find the corresponding split to ${ }^{15}$

$$
u(x, y)=\frac{x\left(x^{2}+y^{2}-a^{2}+\sqrt{c_{1}}\right)}{\sqrt{2 c_{1} \sqrt{c_{1}}+2 c_{1} c_{2}}}
$$

and

$$
v(x, y)=-\frac{y\left(x^{2}+y^{2}+a^{2}-\sqrt{c_{1}}\right)}{\sqrt{2 c_{1} \sqrt{c_{1}}+2 c_{1} c_{2}}},
$$

where $\quad c_{1}=\left((a+x)^{2}+y^{2}\right)\left((a-x)^{2}+y^{2}\right) \quad$ and $c_{2}=x^{2}-y^{2}-a^{2}$. The derivatives of $\phi$ are then given to $^{7}$

$$
\frac{\partial}{\partial z} \phi(z)=-\frac{\sigma_{0}}{8}\left(\frac{\partial}{\partial x} u(x, y)-i \frac{\partial}{\partial y} u(x, y)\right)
$$

and

$$
\frac{\partial}{\partial \bar{z}} \phi(\bar{z})=-\frac{\sigma_{0}}{8}\left(\frac{\partial}{\partial x} u(x, y)+i \frac{\partial}{\partial y} u(x, y)\right) .
$$

The derivatives of the real part, $u(x, y)$, with respect to $x$ and $y$ are then given by

$$
\frac{\partial}{\partial x} u(x, y)=\frac{a^{2}}{\sqrt{2} c_{1}^{2}}\left(\sqrt{c_{1}}-2 c_{2}\right) \sqrt{c_{1}\left(\sqrt{c_{1}}+c_{2}\right)}
$$

and

$$
\begin{aligned}
& \frac{\partial}{\partial y} u(x, y)=\frac{1}{2 \sqrt{2}} \frac{a^{2}}{c_{1}^{2} x y}\left(c_{2}\left(c_{2}-\sqrt{c_{1}}\right)-4 x^{2} y^{2}\right) \\
& \sqrt{c_{1}\left(\sqrt{c_{1}}+c_{2}\right)} .
\end{aligned}
$$

Substitution of these relations back into equations (5) leads the expressions given by (6).

\section{Appendix 2}

Shifting the origin in equation (1) to the left contact edge with $s=x+a$ yields

$$
\begin{aligned}
\sigma_{x y}(s) & =-\frac{2 \sigma_{0}}{4} \frac{s-a}{\sqrt{2 a s-s^{2}}} \\
& =-\frac{\sigma_{0}}{2} \frac{s-a}{\sqrt{2 a} \sqrt{s}} \frac{1}{\sqrt{1-s / 2 a}},
\end{aligned}
$$

and after a binomial expansion of the last square root term

$$
\begin{aligned}
\sigma_{x y}(s) & =-\frac{\sigma_{0}}{2} \frac{s-a}{\sqrt{2 a} \sqrt{s}} \frac{1}{(1-s / 4 a+\ldots)} \\
& \approx \frac{\sigma_{0}}{2} \sqrt{\frac{a}{2}} \frac{1}{\sqrt{s}}-\frac{\sigma_{0}}{2 \sqrt{2 a}} \sqrt{s} \quad \text { for } \quad \frac{s}{4 a} \ll 1 .
\end{aligned}
$$

Close to the contact edge, $s \rightarrow 0$, the first term, being square root singular, will dominate and we drop the second (square root bounded) term for the asymptotic description to find the result stated in equation (7) 


$$
\sigma_{x y}(s)=\frac{\sigma_{0}}{2} \sqrt{\frac{a}{2}} \frac{1}{\sqrt{s}}=K_{I I} \frac{1}{\sqrt{s}} .
$$

For the asymptotic description of the subsurface stress field we start from the negative branch of the complex potential function (2), again with a shift of the origin via $w=z+a$ to the left contact edge

$$
\begin{aligned}
\phi(w) & =-\frac{\sigma_{0}}{8}\left(1+\frac{w-a}{\sqrt{w^{2}-2 a w}}\right) \\
& =-\frac{\sigma_{0}}{8}\left(1+\frac{1}{\sqrt{2 a}} \frac{1}{\sqrt{-w}} \frac{w-a}{\sqrt{1-w / 2 a}}\right) .
\end{aligned}
$$

Again we perform a binomial expansion of the last square root term and find

$$
\begin{aligned}
\phi(w) & =-\frac{\sigma_{0}}{8}\left(1+\frac{1}{\sqrt{2 a}} \frac{1}{\sqrt{-w}} \frac{w-a}{(1-w / 4 a+\ldots)}\right) \\
& \approx-\frac{\sigma_{0}}{8}\left(1+\frac{1}{\sqrt{2 a}} \frac{1}{\sqrt{-w}}(w-a)\right) \quad w \ll 4 a \\
& =\frac{1}{2} \frac{\sigma_{0}}{4} \sqrt{\frac{a}{2}} \frac{1}{\sqrt{-w}}-\frac{\sigma_{0}}{8}+\frac{\sigma_{0}}{8} \frac{1}{\sqrt{2 a}} \sqrt{-w} .
\end{aligned}
$$

Again, dropping the square root bounded term for asymptotic description leads to equation (8)

$$
\phi(w)=\frac{1}{2} \frac{\sigma_{0}}{4} \sqrt{\frac{a}{2}} \frac{1}{\sqrt{-w}}-\frac{\sigma_{0}}{8}=\frac{K_{I I}}{2} \frac{1}{\sqrt{-w}}-\frac{\sigma_{0}}{8} .
$$

